\title{
To Propose a Mobility Based Reconfigurable System under Disaster Management in WSN

\author{
Isha Sharma ${ }^{1}$, Shilpa Gambhir ${ }^{2}$ \\ ${ }^{1,2}$ (Department of Electronics \& Communication, M.M University, Sadopur, Ambala, India)
}

\begin{abstract}
In this work, it proposes mobility based dynamic reconfiguration system in WSN. In this work, it uses the concept of dynamic reconfiguration routing protocol that achieves the need of various applications and also various network conditions. In this work, an environmental data collection scenario is taken. In this, all nodes are in dynamic nature and moves randomly. All nodes are communicating with each other as well as from head nodes. There is a direct communication between head \& nodes. It provides a multi-hop routing based on shortest path in wireless networks. It provides a complete path with the loss of some packets. In this work, a controller is designed which is used to control the movement and location of nodes. Before disaster occurred, all nodes changed their location for security. As disaster under control, there may get back to their locations. But in this, some localization error is present but its value is less than $3 \%$ which is very efficient.
\end{abstract}

Keywords - WSN System, Dynamic Reconfiguration System, Localization, Mobility Management etc.

\section{INTRODUCTION}

A wireless sensor network (WSN) is a self-organized system of small, independent, low cost, low powered and wirelessly communicating nodes dispersed over an area having sink nodes taking the data from sensor nodes and may handle a variety of sensing, actuating, communicating, signal processing, computation, and communication tasks, deployed in the absence of permanent network infrastructure and in environments with limited or no human accessibility. The sink serves as the gateway between the user application and the sensor network. The WSN nodes have no fixed topology, but they can configure themselves to work in such conditions.

MOBILITY [1] in most cases is defined as the movement of nodes physically i.e. transfer a data from sender to receiver across a network without affecting network conditions. The use of personal network provides a new way to mobility i.e. in terms of session and services. In session concept, it defines the data to be delivered to a session without affecting actual existing state. In service mobility concept, it might not be knowledge about location of service. It's just sending and receiving data to and from a user.

The main application of WSN Network is to find the location of nodes which is an important key for both civil and military applications. A dependable locating system is dangerous in many WSN presentations, particularly for mobile ad-hoc networks. During the last decade, many efforts in research have directed on the development of the accuracy of localisation and complication. Dispersed methods using multifaceted filtering and modification procedures such as Kalman filters, Non Belief Propagation as well as other radio mapping methods have been discovered. Among these mapping techniques, Multidimensional Scaling (MDS) and curvilinear analysis are deliberated to be the most efficient.

To design a WSN application, knowledge of many elements of the context is essential as they influence the operation greatly. However, because some aspects of the context are unpredictable and changing, many design choices are based on assumptions and approximations. As an example, consider the case study of a sensor network for structural health monitoring. During the development of such a WSN, it is unknown which influences the nodes might experience, which nodes might crash, and how long exactly the sensor nodes will last with the available energy [4].

Though, in the world of reality, the design of radio communicated indicator at the antenna is neither a round nor a sphere-shaped, and sensors causes the problems which was not valid in any path loss model and the environment of the sensor field. Now a days, these sensor systems are taking antennas which are directional in nature and is due to their benefits such as conservation of energy and better utilisation of bandwidth.

The most common sources of errors are noise and characteristics of channel. In localization system, ranging methods are useful in nature. In this, received signal indicator is mostly used ranging technique. So, the main problem is the proposed work is to find the accurate position of nodes in network. So, a localization algorithm can improve the efficiency of system. 
The rest of paper is ordered as follows. In section II, we discuss the development of WSN in modern era. In Section III, It defines general reconfiguration scheme. In Section IV, it describes proposed work of system. Section V describes the results of proposed system. Finally, conclusion is explained in Section V.

\section{DEVELOPMENT OF WSN}

To design a WSN application, knowledge of many elements of the context is essential as they influence the operation greatly. However, because some aspects of the context are unpredictable and changing, many design choices are based on assumptions and approximations. As an example, consider the case study of a sensor network for structural health monitoring. During the development of such a WSN, it is unknown which influences the nodes might experience, which nodes might crash, and how long exactly the sensor nodes will last with the available energy. Because of these uncertainties, the quality of service of the WSN might develop over time like the solid blue line in Figure 1.

Initially, the WSN will perform well because the context matches the expectations during development. However, at some point $\mathrm{t} 1$ after deployment, a sensor node might have changed position because of some external event, making its sensor readings less accurate and the WSN decrease its performance. Also, another node might experience high interference, resulting in many retransmissions from that node. The higher energy consumption can cause the battery to run out at $\mathrm{t} 2$. If eventually at $\mathrm{t} 3$ the node that aggregates sensor information and transmits it to the base station crashes, the complete WSN will be rendered useless.

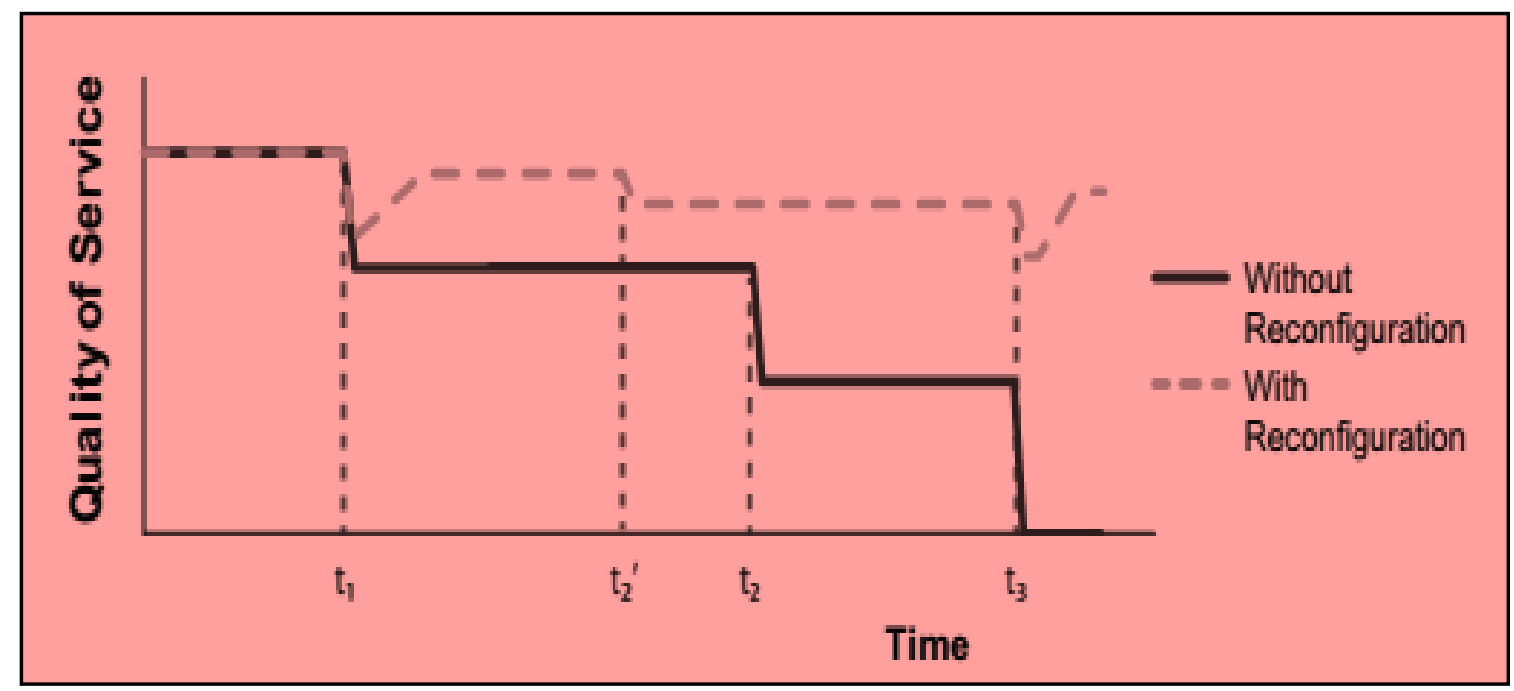

Fig 1: Conceptual Decrease in Quality of Service by Context Changes

If the inherently changing context can be encompassed into the design of the WSN, the application's performance could resemble the course of the dashed green line in Figure 1.5 much more. Whenever (an element of) the context changes, the WSN should be able to detect this and act on it, such that it maintains a suitable configuration even when the context is unpredictable. Going back to the example, the WSN could notice one of its sensors is generating inaccurate results at $\mathrm{t} 1$ and either adjust its sensitivity or disable its sensor to prevent incorrect readings. At $\mathrm{t} 2$, the node that is experiencing much interference could detect the higher energy demand. By increasing the length of its duty cycle, it can avoid completely running out of energy at $t 2$, with only a small decrease in performance. Additionally, if a component providing important functionality crashes, the functionality could be transferred to another node, in which case the WSN could continue to run after $\mathrm{t} 3$. In this way, a developer does not have to determine the exact configuration, sensor accuracy or other properties at design time. Instead, he or she can delegate these choices to the network, and let them be determined at run-time.

For these and many other scenarios, reconfiguration can increase the lifetime and suitability of a WSN in a changing context. However, because of the limited resources of the nodes, WSN deployments are often tailored to specific application requirements in order to get the maximum performance possible. This results in a large variety of application-specific components. As an extension to these components, reconfiguration could also take many forms, tailored specifically to each application. However, when the application-specific elements can 
be isolated, a generalized middleware can be constructed that provides a uniform solution for reconfiguring many different applications.

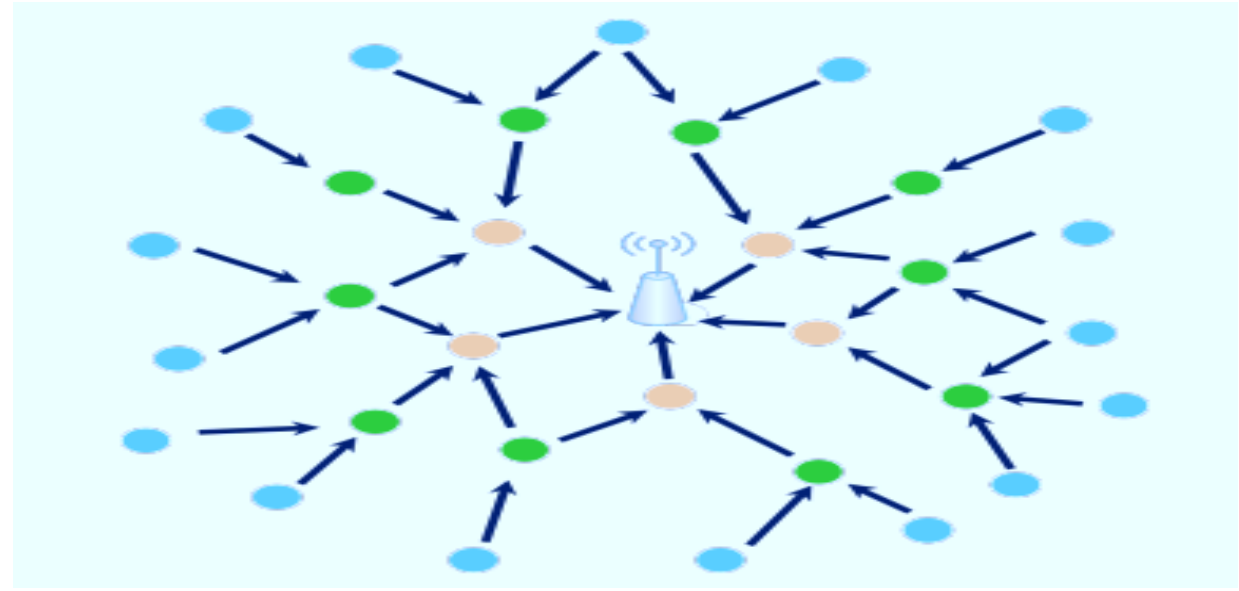

Fig 2: Node Traffic in WSN [3]

\section{RECONFIGURATION MIDDLEWARE FOR WSN}

Earlier research efforts for WSN middleware have focused on various goals, ranging from simplifying localized data sharing between nodes to providing a complete and generic data collection platform. Reconfiguration can be performed in several ways. One solution is to monitor the WSN's quality of service at the base station and reprogram (parts of) the sensor nodes when necessary. An alternative is to locally monitor the context and perform reconfiguration on each node individually.

\section{A. Centralized Reconfiguration}

From survey, some authors detect the need for reconfiguration of a WSN by creating a global model based on information coming from sensor nodes at run-time. Next, a design space search is performed in order to come up with a suitable new configuration, which is subsequently transferred on to the sensor nodes. However, because the tool chain of the dominant WSN operating system, Tiny OS, compiles software components to a static image, this requires replacing the entire code image after deployment. This uses much bandwidth and energy. Some provides a method for creating virtual machines for sensor nodes that execute small script-like programs [12]. These scripts can be sent to a node and loaded and unloaded at run-time. Although this approach is more efficient than reprogramming, a disadvantage is that applications are required to be expressed as a list of generic operations, with little room for specialized functionality.

\section{B. Distributed Reconfiguration}

A solution, in which nodes reconfigure themselves without a centralized algorithm, is Impala [5]. Each node contains an application adapter and update component. These respond to certain events in the network and periodically check system and application parameters. Software components are modelled by a finite state machine. If specific preconditions are satisfied, the application is transferred to a different state. A downside of this approach is its granularity: an application can only have a limited number of states, and even small changes take up a complete state. Also, little attention is paid to the reasoning on reconfiguration, and the authors have not yet succeeded in implementing it on a sensor node platform.

Here, nodes publish their functionality as a service to which other nodes can subscribe. When an event in the network or the context decreases the functionality that a specific node offers, the subscribed nodes try to find other nodes with a better service level for the required functionality. However, in sparse networks where there are few alternative nodes with certain functionality, the application can still fail, since these solutions do not consider changing the nodes themselves, but only the relations between them.

\section{Clustering Approach}

In WSN, clustering has become a developing expertise for energy balanced applications. In this, they developed a detection of failure method using cluster based communication for providing accuracy and completeness in 
system. In this system, it divides the whole network into various clusters and then spread out into individual areas or region. Intra-cluster heartbeat diffusion is accepted to classify unsuccessful nodes in each bunch.

\section{Reconfigure Intelligence}

Achieving automatic reconfiguration requires some intelligent component to reason about when to change which components. In the larger area of computer science, artificial intelligence has been a research topic for many years and has branched into many subcategories. Some of these can be interesting to apply in the context of wireless sensor networks. When considering WSNs that reconfigure according to their context, one solution could be to treat the reconfiguration as a multi-parameter optimization problem.

An alternative solution is to use a heuristics based approach like expert systems, to reason over reconfiguration. In expert systems, the necessary information is represented by facts in a database. A repository of rules encapsulates knowledge about the system and is used to infer new information or determine which action is suitable. In FACTS these constructs are used to support an event-condition action based middleware. DSN uses similar constructs, but only to simplify WSN development by creating a new declarative programming language. Although the authors argue that it simplifies WSN development, the solution is used to define standard node behaviour, but both solutions do now allow reconfiguring the node's application depending on its context.

\section{PROPOSED RECONFIGURATION SCHEME}

An environmental collection of data means a scientist is willing to gather various readings of sensors from a collection of points in surroundings along a span of time. This is used to find trends of data in environment. In this work, scientist or user wants collection of data from various spread points from area provided and then data analysed is done offline. The main requirement in this work is to collect large data at regular interval of time and detecting the nodes location at each period of time.

The environmental data collection is considered by large network area having large no. of nodes which are transmitting data and also sensing the locations and other data collection. Then they transmit data back to base station so that collected data will be stored. In this, these nodes does not require high data rates for transmission. They have long lifetime and generally low data rates. In network mode, these nodes are dispersed over an environment. These are placed randomly in area provided and also the distance between them is also minimum so that performance of network will be useful.

After placement of nodes, it requires to determine the topology of network i.e. tree or star based etc. Then provide optimum routing technique based on shortest path so that delay will be minimized. In this routing, data is routed through various nodes so that data may reach to destination. In environmental collection of data, it does not require the development of optimal routing by own but it requires the routing strategy outside network and communication is also necessary. This is probable by creating the physical topology of the net is comparatively continual.

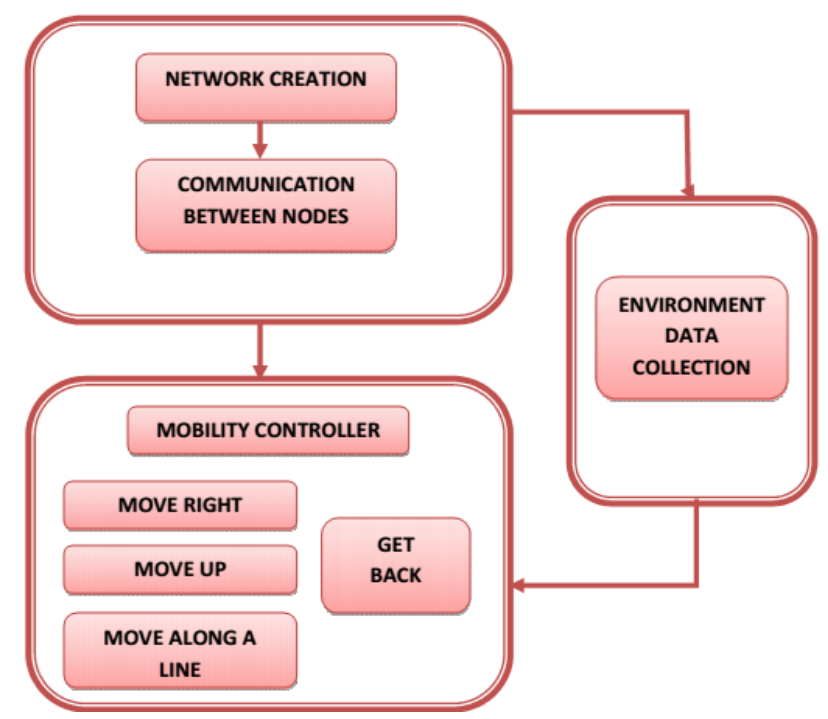

Fig 3: Proposed System Model 
Another feature of WSN application is security monitoring. In this work, network is containing nodes which are placed in outdoor environment and also provided at fixed locations. They work as monitoring nodes that work continuously. In security work, these

nodes only monitor the data but does not collect the data which is done is environmental data monitoring. Each node continuously checks the status of sensors but it transmit a report of data when there is security problem in its area. It requires the use of alarm messages which must be reliable in nature.

Currently, it is hard to configure the service of routing in a large area sensor network because it provides a preconfigured network service with each node statically. So, it suggests a mobility based network reconfiguration system in WSN which can be dynamically reconfigured. Then it provides the mechanism of dynamic reconfiguration. The dynamic reconfiguration at node level sought to minimize energy consumption by dynamically adjusting hardware platforms of sensor nodes. The utilization of reconfiguration technique have to consider dynamic factors, such as changes in user requirements, variations in communication channel quality, application changes etc.

The main objective of this work is to design mobility based self-network reconfiguration system in WSN. The next objective is to use dynamically reconfigurable routing protocol with shortest path for routing in network (shown in fig 3). Environmental observation and forecasting may include eruption warning system, flood detection, study of earthquake cyclone and tsunami warning system, water quality monitoring etc.

A good warning system can help to avoid the damages produced by expected disasters. Sensor nodes can be used to provide the circumstances of plants and animals in wild environment, as well as the ecological constraints of the habitat. Sensor can be deployed under water or on the ground to monitor the quality of air and water. Monitoring of Air quality can be used for air pollution control and water quality monitoring can be used in biochemistry field. Sensors can also be deployed to detect natural or non-natural disasters. For example, sensor nodes deployed in a forest can also detect the exact origin of the fire before the fire is spread uncontrollable. Seismic sensors can be used to notice the route and magnitude of earthquakes.

In above figure, the first step describes the sensors are being deployed in a disaster area. Sensors are randomly spread over the area. Each sensor has a sensor ID shown along with it. It will be used to address any sensor throughout the process. Here we take large number of sensors so that proposed scheme will evaluate easily. No two nodes overlap each other. The environmental data collection is considered by large network area having large no. of nodes which are transmitting data and also sensing the locations and other data collection. Then they transmit data back to base station so that collected data will be stored. In this, these nodes does not require high data rates for transmission. Then provide random mobility in nodes to show that all nodes are dynamic in nature. All nodes are communicating with each other on the basis of shortest path calculated.

After the deployment of the sensor nodes, there is a Head node selection by polling method. Then head check the status of each node and collects the environmental data from sensor nodes. For this, there is a direct communication between head \& nodes. Head asks the nodes about environment conditions, then reply back to head about status. Now if temperature goes above threshold due to any disaster effect, the nodes sense data and tells to the head and starts moving from their locations. Then they collect to any other location and when the disaster under control then head orders the nodes to repositioning or reconfigure their locations within minimum time.

\section{RESULTS \& DISCUSSION}

Initial configuration of network is setup and then each node senses the data from sensors and transmits data back to base station so that collected data will be stored. In this, these nodes does not require high data rates for transmission. In this work, we are taking the scenario of 60 nodes which are placed randomly in network. After placement of nodes, it requires to determine the topology of network i.e. tree or star based etc. each sensor node has its ID with it so that they can find out other node in network. Then provide optimum routing technique based on shortest path so that delay will be minimized. 


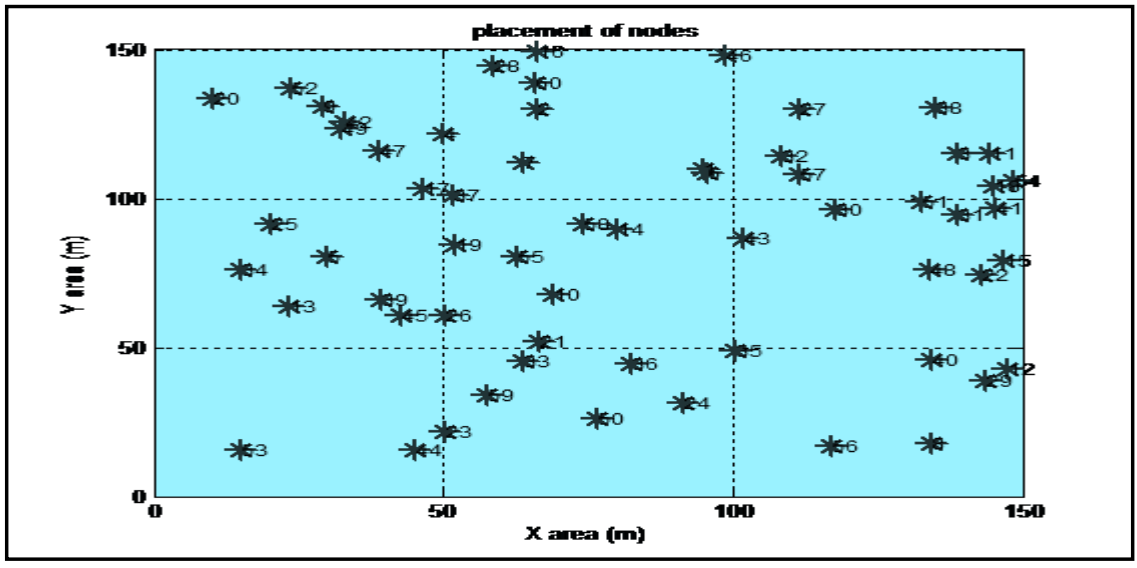

Fig 4: Network Creation in WSN

In this routing, data is routed through various nodes so that data may reach to destination. In above figure, the first step describes the sensors are being deployed in a disaster area. Sensors are randomly spread over the area. Each sensor has a sensor ID shown along with it. It will be used to address any sensor throughout the process.

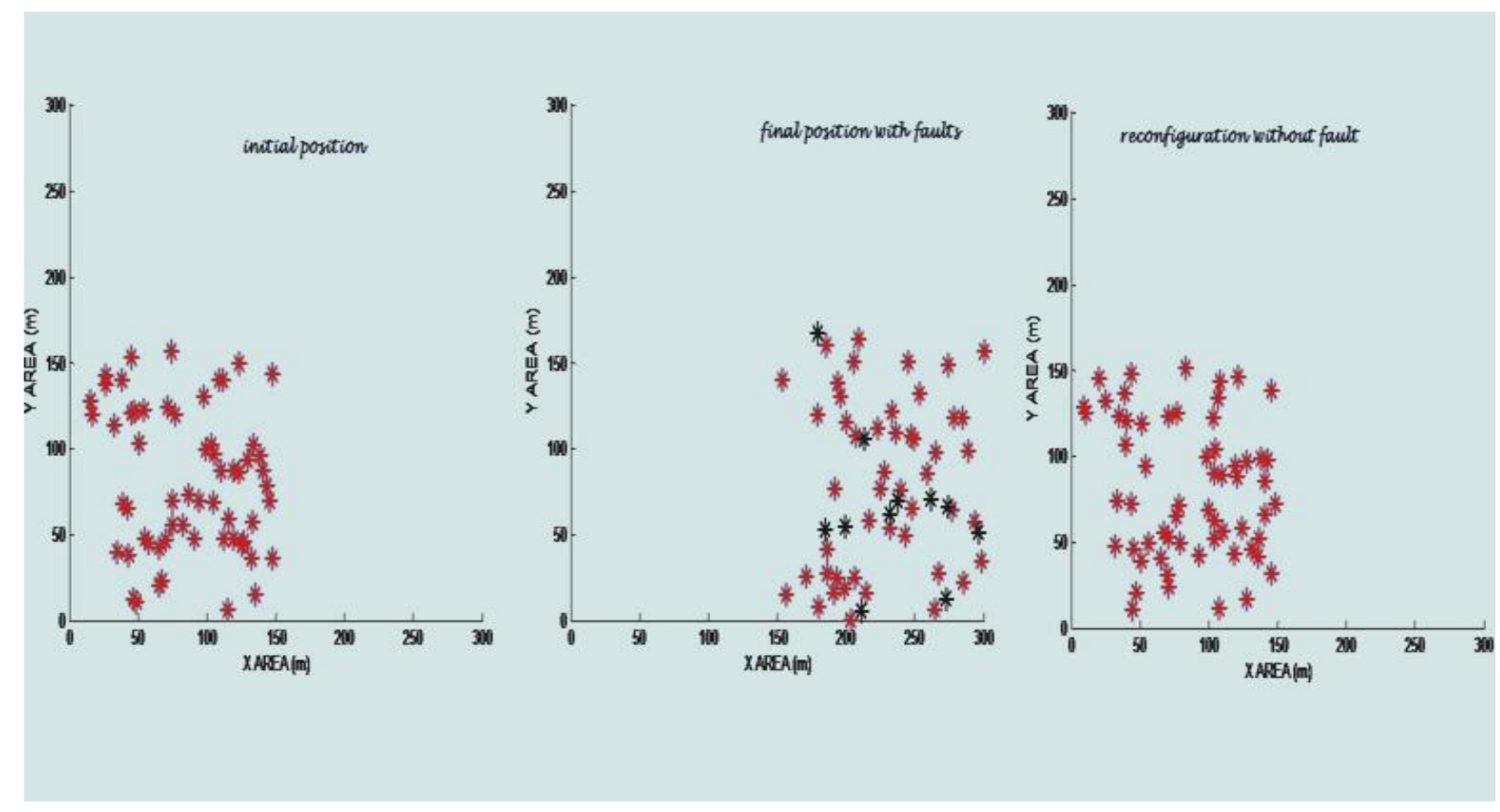

Fig 5: Network Reconfiguration Scenario in Proposed System

Here we take large number of sensors so that proposed scheme will evaluate easily. No two nodes overlap each other. They provide a topology initially which is random in nature. Reconfiguration means the process of transition of network nodes from one point to another transition in space. In this work, it requires two operations. First is to find the new location with new configuration and secondly it requires the operation so that network will be reconfigured. Both tasks are performed by a controller designed.

Here a controller is used to control the movement of nodes and its location. It provides the new location or area where network is to be configured. In figure 5, initially network is placed in area of $150 * 150 \mathrm{~m}^{2}$ randomly. Each node is mobile in nature and controlled by a controller used. After some time, controller changes its topology and updates its location to a new location. While updating the nodes location, some nodes lost its energy and becomes faulty nodes. During reconfigure time, each faulty node is replaced by a new one so that network may work better. 


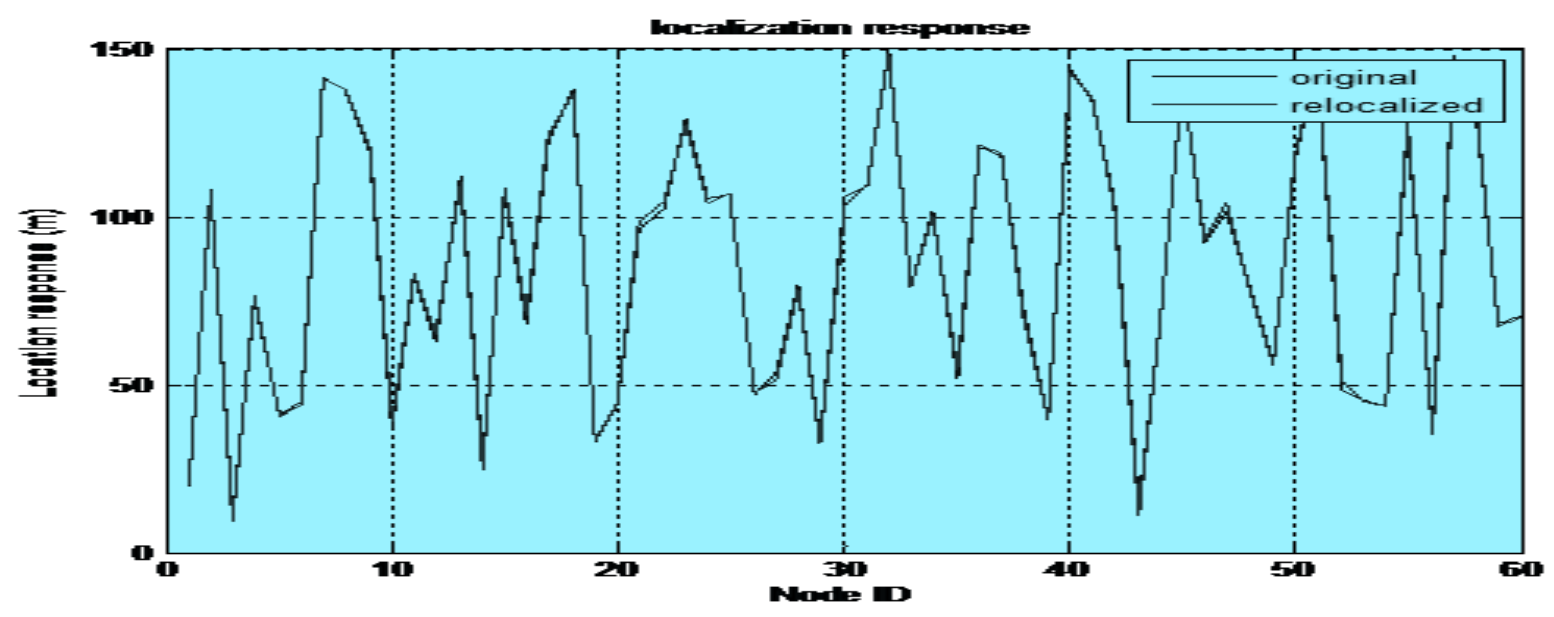

Fig 6: Localization Response of Proposed System

It is hard to find out or checking the operating conditions of sensor network. During hardware reconfigure system, it requires the proper hardware requirement with suitable environment so that it works better. Due to this, there is a need of software reconfigure system for sensing network.

The proposed protocol is proved steady by our truthful experimentation. Moreover, the experiment results validate that proposed protocol is with the ability of dynamic reconfiguration when it is running on the WSN platform. The main parameter is the localization error of nodes. As nodes are moving and they changed their location when disaster occurred. So, when all nodes get back to their location after disaster control, there have some localization error but this value must be minimum for better response.

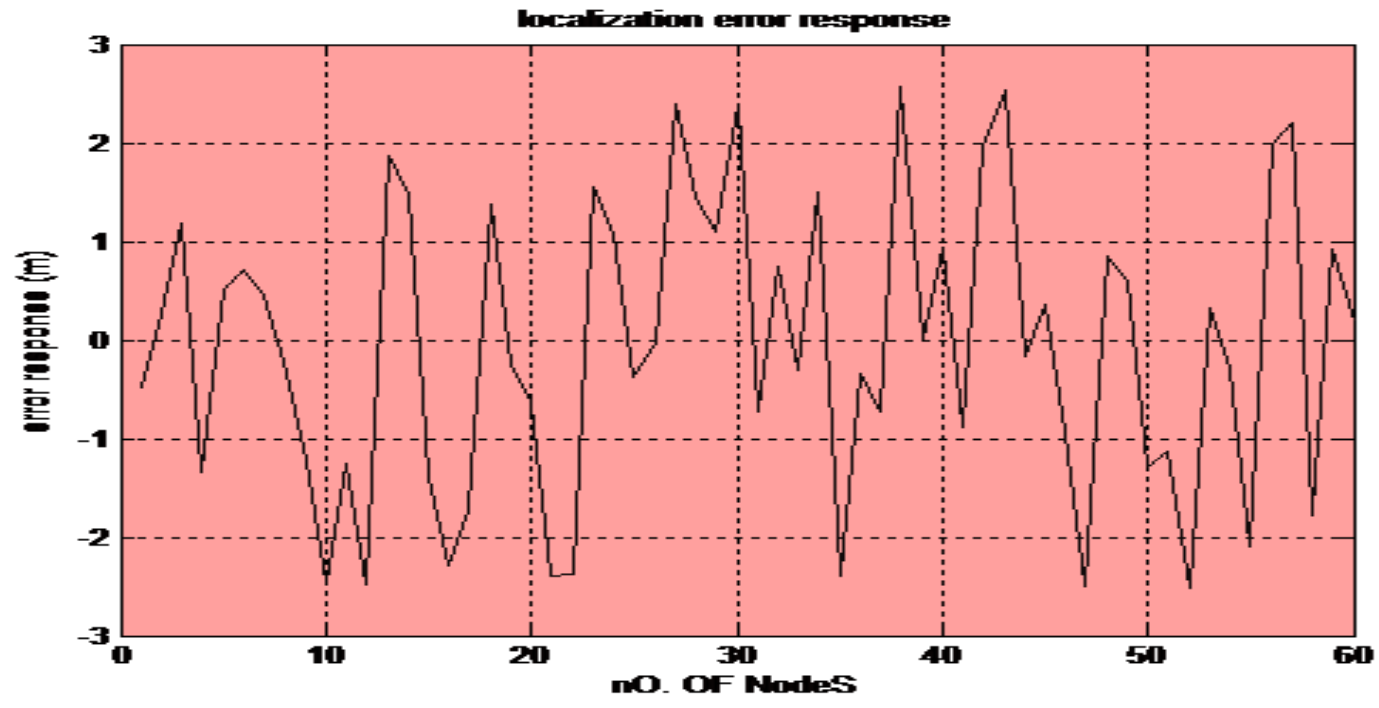

Fig 7: Localization Error of Proposed System

\section{CONCLUSION}

This work provides a technique for reconfiguration of network nodes in WSN with the help of controller system. All scenarios of the dynamic reconfiguration infrastructure have been evaluated. In this work, all nodes are communicating with each other. A head is provided for giving the instructions to all nodes. In this, it takes the scenario of disaster in forests. Before disaster occurred, all nodes changed their location for security. As disaster under control, there may get back to their locations. Each node is mobile in nature and controlled by a controller used. After some time, controller changes its topology and updates its location to a new location. While updating the nodes location, some nodes lost its energy and becomes faulty nodes. During reconfigure time, each faulty node is replaced by a new one so that network may work better. But in this, some localization error is present but its value is less than $3 \%$ which is very efficient. 


\section{REFFERENCES}

[1] Rappaport, Wireless Communications, 2nd edition. Singapore: Pearson Education, Inc., 2002.

[2] Davis. Basics of Design: Battery Power Management. Supplement to Electronic Design.2004

[3] S. Gao,Y. Piao, “DRRP: A Dynamically Reconfigurable Routing Protocol for WSN”, IEEE 2014.

[4] M. Mwila, K. Djouani, "An Well-organized Approach to Node Localisation and Tracking in Wireless Sensor Networks", IEEE Ad Hoc and Sensor Networking Symposium, 2014.

[5] F. Nazir, R. Boreli, Stephen Herborn, "A Case Study for Mobility Management Protocol Co-existence", IEEE 2008.

[6] T. Condeixa, Susana Sargento, "Decoupling and Distribution of Mobility Management", IEEE International Workshop on Mobility Management in the Networks, 2011.

[7] A. Majumder, Sudipta Roy, Kishore Kumar Dhar, "Design and Analysis of an Adaptive Mobility Management Scheme for Handling Internet Traffic in Wireless Mesh Network", IEEE International Conference on Microelectronics, Communication and Renewable Energy, 2014.

[8] Jong-Hyouk Lee, Jean-Marie Bonnin, "Comparative Handover Performance Analysis of IPv6 Mobility Management Protocols", IEEE Transactions On Industrial Electronics, Vol. 60, No. 3, March2012.

[9] Y. E. Krasteva, J. Portilla, J. M. Carnicer, "Remote HW-SW Reconfigurable Wireless Sensor Nodes", IEEE 2008.

[10] A. Abbagnale, Emanuele Cipollone, "A case study for evaluating IEEE 802.15.4 wireless sensor network formation with mobile sinks", IEEE 2008.

[11] Reed Tompkins, Thomas B. Jones, "Reconfiguration and Management in Wireless Sensor Networks", IEEE 2013.

[12] Szczodrak, Omprakash Gnawali, "Dynamic Reconfiguration of Wireless Sensor Networks to Support Heterogeneous Applications", IEEE International Conference on Distributed Computing in Sensor Systems, 2013.

[13] Marco, F. Gallo, "Implementing Adaptation and Reconfiguration Strategies in Heterogeneous WSN", IEEE 27th International Conference on Advanced Information Networking and Applications, 2013.

[14] Fei Ding, Kenneth A. Loparo, "Hierarchical Decentralized Network Reconfiguration for Smart Distribution Systems-Part II: Applications to Test Systems", IEEE Transactions on Power Systems, Vol. 30, No. 2, March 2015.

[15] Shilpa Gambhir, "Comparative Study of Various Routing Protocols for Wireless Sensor Networks”, IJRASET, Vol. 3, Issue 12, December 2015.

[16] Jean A. Guevara, Enrique A. Vargas, "Dynamically Reconfigurable WSN Node Based on ISO/IEC/IEEE 21451 TEDS", IEEE Sensors Journal, Vol. 15, No. 5, May 2015.

[17] Wonho Kang and Youngnam Han, "SmartPDR: Smartphone-Based Pedestrian Dead Reckoning for Indoor Localization", IEEE Sensors Journal, Vol. 15, No. 5, May 2015. 